\title{
Avaliação da perimetria macular em pacientes com degeneração macular relacionada à idade por meio do oftalmoscópio de rastreamento a laser
}

\author{
Evaluation of macular perimetry in patients with age-related macular \\ degeneration using the scanning laser ophthalmoscope
}

\author{
Lúcio Moraes ${ }^{1}$ \\ Ann E. Elsner ${ }^{2}$ \\ Christian Kunze $^{3}$ \\ Hisashi Suzuki ${ }^{4}$ \\ Márcio Bittar Nehemy ${ }^{5}$ \\ DaniloSoneSoriano $^{6}$ \\ Newton Kara-José ${ }^{7}$
}

Trabalho realizado na Harvard Medical School e na Faculdade de Medicina da Universidade de São Paulo. Pós-graduando do Departamento de Oftalmologia da Faculdade de Medicina da Universidade de São Paulo USP - São Paulo (SP) - Brasil.

2 Doutora, Professora da Harvard Medical School, Boston -USA.

${ }^{3}$ Professor do Departamento de Oftalmologia do Innsbruck Medical University - Áustria.

${ }^{4}$ Doutor, Professor da USP - São Paulo (SP) - Brasil.

${ }^{5}$ Professor, Chefe do Serviço de Retina e Vítreo da Faculdade de Medicina da Universidade Federal de Minas Gerais - UFMG - Belo Horizonte (MG) - Brasil. Doutor, Professor da USP - São Paulo (SP) - Brasil.

${ }^{7}$ Livre Docente, Professor Titular do Departamento de Oftalmologia da USP - São Paulo (SP) - Brasil e da Universidade de Campinas - UNICAMP - Campinas (SP) - Brasil.

Endereço para correspondência: Instituto Panamericano da Visão. Av. T-8, 171 - Setor Marista - Goiânia (GO) CEP 74210-270

E-mail: luciomoraes@ipvisao.com.br

Recebido para publicação em 03.05.2006

Última versão recebida em 16.03.2007

Aprovação em 11.06.2007

Resumo da primeira parte da Tese de Doutorado apresentada ao Departamento de Oftalmologia, Faculdade de Medicina da USP.

Esta pesquisa teve apoio da Fundação de Amparo à Pesquisa do Estado de São Paulo (FAPESP)

Nota Editorial: Depois de concluída a análise do artigo sob sigilo editorial e com a anuência da Dra. Áisa haidar Lani sobre a divulgação de seu nome como revisor, agradecemos sua participação neste processo.

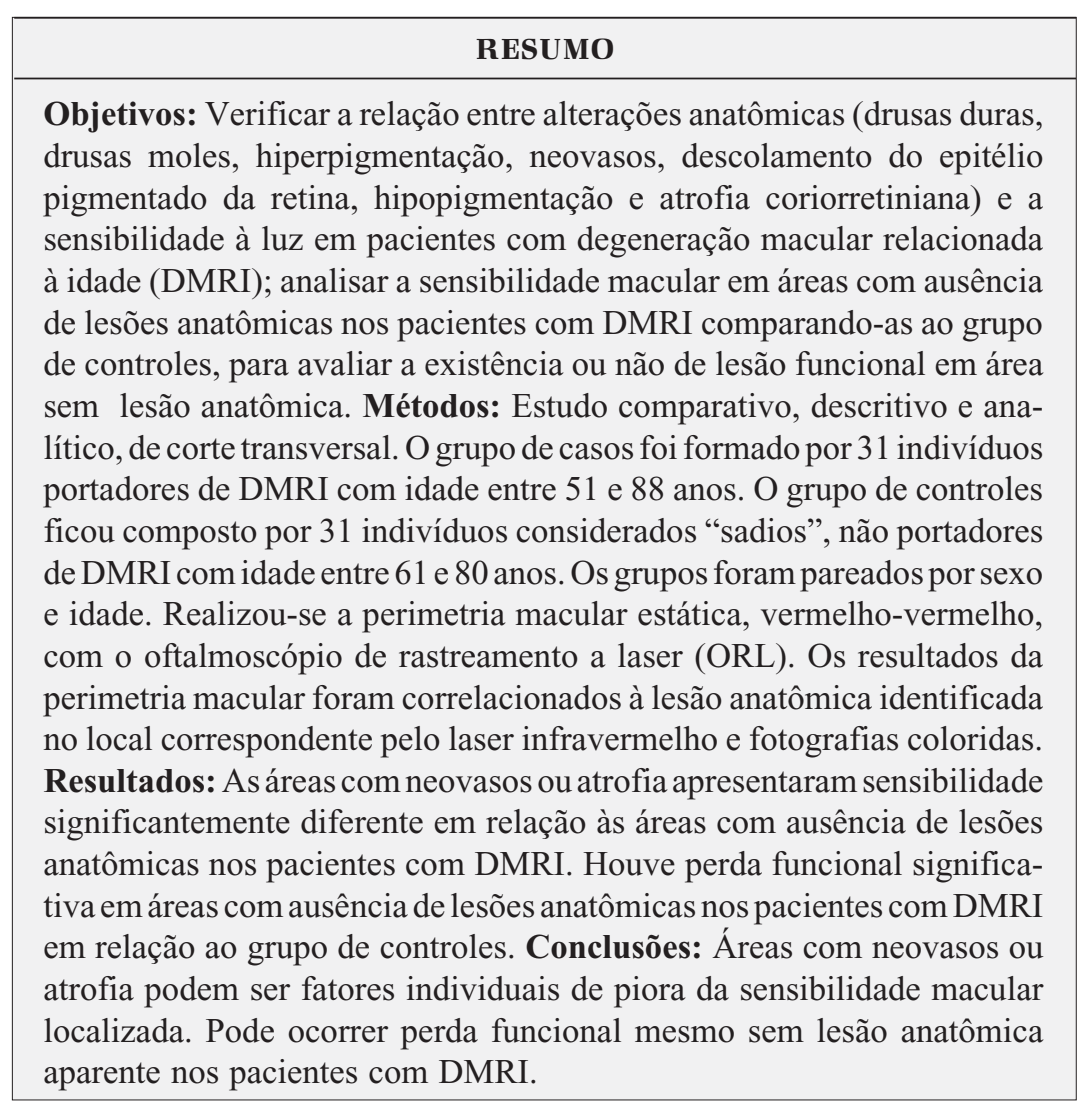

Descritores: Perimetria; Oftalmoscópios; Degeneração macular; Neovascularização coroidal; Fatores etários

\section{INTRODUÇ̃̃O}

Para melhorar o entendimento do prognóstico visual a partir dos achados clínicos, é fundamental que se relacione a aparência do fundo ocular com a função visual na doença macular relacionada à idade.

A medida de função macular semelhante em áreas com e sem determinada lesão anatômica sugere que tal lesão não seja o agente causal de perda de função localizada na retina.

A identificação das lesões anatômicas relacionadas com a perda de sensibilidade em um ponto específico da retina pode indicar que a perda 
da função macular focal seja um sinal precoce do aparecimento destas lesões anatômicas.

A diminuição da sensibilidade foveal pode predizer o desenvolvimento das formas avançadas de DMRI ${ }^{(1)}$. Portanto, a mudança de sensibilidade poderia servir de alerta ao oftalmologista para monitorar o paciente, neste local específico, com o objetivo de identificar e tratar precocemente uma lesão anatômica no início de seu desenvolvimento.

O diagnóstico oportuno das alterações na estrutura normal e na função pode possibilitar o tratamento precoce, evitando que o dano se amplie. As medidas preventivas e terapêuticas somente são efetivas quando adotadas antes que ocorram danos irreversíveis aos fotorreceptores.

Os estudos têm ressaltado mais os aspectos anatômicos da DMRI pela dificuldade de avaliação funcional com os aparelhos até então disponíveis.

Para melhor avaliar o quanto e onde está afetada a função retiniana foi desenvolvida a perimetria retiniana pelo oftalmoscópio de rastreamento a laser (ORL), único instrumento disponível atualmente que permite a precisa correlação anatômico-funcional na área macular. Utilizando o ORL, o examinador vê o estímulo visual testado diretamente na retina do paciente, podendo estabelecer a relação precisa entre a função visual e o aspecto anatômico nas doenças maculares. Essa informação pode ser útil na avaliação das doenças maculares, bem como na conduta de seu tratamento ${ }^{(2-4)}$.

Os objetivos do presente estudo são:

1) verificar a relação entre alterações anatômicas específicas (drusas duras, drusas moles, hiperpigmentação, descolamento do epitélio pigmentado da retina, hipopigmentação, neovasos e atrofia coriorretiniana) e a sensibilidade macular;

2) analisar a sensibilidade macular em áreas com ausência de lesões anatômicas nos pacientes com DMRI, para avaliar a existência ou não de lesão funcional em área sem lesão anatômica.

\section{MÉTODOS}

Foi realizado um estudo comparativo, descritivo e analítico, de corte transversal no período de janeiro de 1997 a junho de 2002 .

Todos os participantes foram devidamente informados sobre os objetivos do trabalho e sobre os procedimentos a que seriam submetidos e assinaram termo de consentimento livre e esclarecido, em pesquisa aprovada pelo Comitê de Ética da Universidade de São Paulo.

A população amostral foi composta por um grupo de pacientes com DMRI diagnosticada clinicamente e documentada por fotografia colorida e método de imagem pelo laser infravermelho, denominado grupo de casos. Formou-se outro grupo de indivíduos, considerados "sadios", ou sem evidência de DMRI ao exame oftalmológico clínico, pareados em função de sexo e idade com o grupo de casos, denominados grupo de controles. Todos os indivíduos dos dois grupos foram examinados por dois especialistas em retina, que se utilizaram de biomicroscopia com lâmpada de fenda, oftalmoscopia indireta e tonometria.

Consideraram-se como critérios de inclusão: idade igual ou superior a 50 anos; diagnóstico clínico de DMRI; entendimento e assinatura do termo de consentimento livre e esclarecido; serem pacientes alertas, cooperativos, com boa coordenação motora manual; padrão de fixação nível 2 (apresenta um local de preferência para fixação na retina, apesar de algumas perdas transitórias) ou fixação nível 3 (fixação estável), conforme padrões estabelecidos por Acosta et al. ${ }^{(5)}$.

Foram considerados critérios de exclusão: ametropia maior que seis graus; deficiência de visão de cores; doenças oculares que pudessem afetar o campo visual (glaucoma, retinocoroidopatia serosa central, oclusão de vaso retiniano, diabetes melito, neuropatia óptica isquêmica, rotura de coróide e outras), pacientes em uso de drogas que podem afetar a função visual (cloroquina e outras), trauma ocular prévio, doenças desmielinizantes, olhos que foram tratados com laser previamente, cirurgia intraocular prévia, outra que não catarata, e relato de história de contra-indicação para o uso de midriáticos.

O grupo de casos foi formado por 31 indivíduos (31 olhos) portadores de DMRI, leucodérmicos, sendo 20 mulheres e 11 homens, com idade entre 51 e 88 anos (média de idade 73,6 anos, com desvio padrão de 8 anos).

O grupo de controles ficou composto por 31 indivíduos (31 olhos) não portadores de DMRI, leucodérmicos, sendo 20 mulheres e 11 homens, com idade entre 61 e 80 anos (média de idade 70,3 anos e desvio padrão de 5 anos).

Utilizou-se a perimetria macular por meio do ORL para quantificar a sensibilidade à luz dos fotorreceptores (cones e bastonetes) em 12 localizações da área macular.

Antes de cada exame de perimetria, ajustou-se a intensidade de todas as fontes de laser, para obterem-se condições padronizadas de iluminação. As calibrações foram feitas utilizando um fotômetro (EG\&G,DR2550; Gamma Scientific ${ }^{\circledR}$ San Diego, Califórnia-EUA), calibrando-se cada fonte de laser separadamente.

No início do exame, o paciente era orientado a sentar-se em frente ao ORL, apoiando o queixo no suporte próprio do aparelho, de modo que um dos olhos ficasse alinhado com as luzes de laser vindas de dentro do aparelho. O paciente então visibilizava o fundo vermelho do aparelho e, no centro, uma mira, que servia como ponto de referência para fixação de seu olhar. $\mathrm{O}$ examinador visibilizava a área macular e a mira em um monitor de computador. Iniciava-se a apresentação dos estímulos luminosos na área macular. Caso o paciente visibilizasse o estímulo, apertava um botão que estava em sua mão. A cada apresentação de estímulo, o examinador posicionava o cursor do computador em um mesmo ponto de referência anatômico na imagem do fundo de olho que estava em seu monitor, como, por exemplo, a bifurcação de um vaso. Através dessa manobra um sistema computadorizado de seguimento de imagens garantia o posicionamento do estímulo nos locais selecionados da área macular (Figura 1).

$\mathrm{O}$ intervalo de intensidade luminosa dos estímulos (cal- 
culado pela intensidade de luz máxima possível de ser gerada com um estímulo à retina menos a intensidade luminosa básica presente no fundo do aparelho) utilizado foi de 2,5 unidades logarítmicas (log) ou $25 \mathrm{~dB}$ acima da iluminação de fundo. Logo, o resultado da perimetria para cada ponto testado pôde variar de zero $\mathrm{dB}$ a $25 \mathrm{~dB}$. Para referência, $1 \mathrm{~dB}$ representa a atenuação de 0,1 unidade $\log$ da intensidade luminosa máxima. Os valores de sensibilidade medidos em $\mathrm{dB}$ indicam a quantidade de filtros de densidade neutra interpostos no trajeto da luz de estímulo. Portanto, valor alto de sensibilidade em $\mathrm{dB}$ indica que foi usado filtro mais denso para atenuação da luz e que o paciente tem melhor sensibilidade, pois conseguiu detectar esse estímulo luminoso fraco.

Foi realizada perimetria estática com estímulos luminosos vermelhos e iluminação básica do fundo do aparelho de ORL também vermelha. Todos os exames de perimetria foram realizados pelo mesmo pesquisador (LM). O laser Hélio Neônio (HeNe $633 \mathrm{~nm}$ ) foi utilizado para a apresentação dos estímulos, para iluminação básica do fundo do ORL (500 td) e para formação da mira como ponto de referência. O laser IV gerava a imagem da mácula no monitor do examinador.

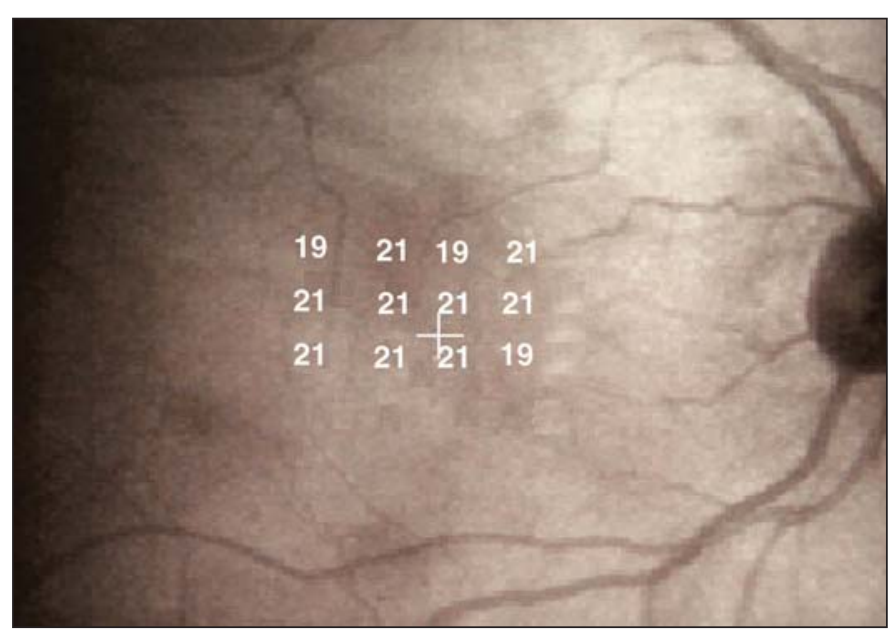

Figura 1 - Avaliação anatômico-funcional da mácula. Os números representam a sensibilidade macular em decibéis (dB).
Em cada sessão de perimetria macular, 12 localizações foram testadas aleatória e automaticamente por um sistema supralimiar progressivo, com estímulos de forma quadrangular e tamanho de $0,4^{\circ} \times 0,4^{\circ}$, tamanho aproximado do Goldmann III, 120 x $120 \mathrm{~mm}$ na retina, 200 milissegundos de duração, apresentados em flashes nos 10 graus centrais maculares. Se o paciente percebesse o estímulo, ocorria uma redução progressiva de 0,15 unidades logarítmicas $(1,5 \mathrm{~dB})$ na intensidade luminosa deste, e, se o estímulo não fosse percebido, gerava-se um último estímulo com intensidade luminosa aumentada em 0,08 unidades logarítmicas $(0,8 \mathrm{~dB})$. A avaliação finaliza-se neste ponto. Utilizou-se a versão 3.0 do programa para perimetria AACHEN ${ }^{\circledR}$, Aachen, Alemanha, na apresentação dos estímulos, coleta de respostas e computação da sensibilidade no ORL.

Foram realizados dois testes de perimetria nos doze locais selecionados na área macular de cada indivíduo. Posteriormente, foi computada a média para cada um desses locais, em cada paciente. A duração de cada exame de perimetria foi de sete a dez minutos. Um intervalo de 30 minutos entre os testes minimizou o efeito da fadiga.

Quantificou-se o número e qualificou-se o tipo de estrutura sub-retiniana presente nos 10 graus centrais $(3.000 \mu \mathrm{m})$, nos 12 locais testados de sensibilidade retiniana, baseado nas imagens do laser infravermelho (830 $\mathrm{nm}$ de comprimento de onda e potência de $50 \mu \mathrm{W} / \mathrm{cm}^{2}$ ) e nas fotos coloridas (slides de 35 milímetros). Alterações anatômicas, como drusas, hiperpigmentação, neovasos e outras, foram registradas como presentes ou ausentes no local testado pela perimetria macular. Considerou-se como presente a lesão que ocupasse mais de 50\% da área total do estímulo do exame de perimetria.

Adotou-se, em todos os testes estatísticos realizados, o nível de significância de $0,05(\alpha=5 \%)$.

\section{RESULTADOS}

A tabela 1 evidencia que a hiperpigmentação foi a lesão anatômica com maior média de sensibilidade $(18,88 \mathrm{~dB})$ e menor desvio padrão $(1,13 \mathrm{~dB})$. Hipopigmentação e atrofia

\begin{tabular}{|c|c|c|c|c|c|}
\hline Variáveis & $\mathbf{n}$ & Média (dB) & DP (dB) & Mínimo (dB) & Máximo (dB) \\
\hline Ausência de lesões anatômicas & 13 & 18,81 & 1,55 & 17,00 & 21,50 \\
\hline Drusas duras & 15 & 17,87 & 1,64 & 15,00 & 21,00 \\
\hline Drusas moles & 9 & 17,78 & 3,27 & 11,00 & 21,00 \\
\hline Hiperpigmentação & 8 & 18,88 & 1,13 & 17,00 & 21,00 \\
\hline Drusas duras + hiperpigmentação & 18 & 18,03 & 2,23 & 14,00 & 22,50 \\
\hline Drusas moles + hiperpigmentação & 13 & 17,35 & 2,98 & 9,50 & 21,00 \\
\hline Neovasos & 7 & 14,29 & 6,61 & 4,50 & 19,00 \\
\hline DEP & 4 & 15,50 & 3,11 & 11,00 & 18,00 \\
\hline Neovasos + DEP & 2 & 14,50 & 3,54 & 12,00 & 17,00 \\
\hline Hipopigmentação e atrofia coriorretiniana & 8 & 9,63 & 6,82 & 0,00 & 18,00 \\
\hline
\end{tabular}


foram as lesões com menor média de sensibilidade $(9,63 \mathrm{~dB})$ e maior desvio padrão $(6,82 \mathrm{~dB})$.

A tabela 2 mostra que existe diferença entre as médias de sensibilidade nas formas não exsudativa e exsudativa $(\mathrm{p}<0,001)$. Olhos com exsudação apresentam, em média, pior sensibilidade em relação aos olhos sem exsudação. A forma exsudativa apresenta maior coeficiente de variação $(39 \%)$ do que a forma não exsudativa (13\%).

Utilizando-se os dados do grupo de casos descritos na tabela 1, formou-se a tabela 3, que mostra neovasos e atrofia como as únicas lesões com diferença significativa em relação às áreas aparentemente normais no grupo de casos. Ambas as lesões apresentaram média de sensibilidade significativamente inferior à média da sensibilidade das áreas com ausência de lesões anatômicas no grupo de casos.

Existe diferença significativa entre a média de sensibilidade de todos os locais testados no grupo de controles e a média de sensibilidade dos locais com ausência de lesões anatômicas do grupo de casos $(\mathrm{p}<0,001)$ (Tabela 4). Os locais supostamente normais pela aparência clínica no grupo de casos, na realidade, já apresentam diminuição da sensibilidade, portanto não são de fato normais.

\section{DISCUSSÃO}

Os resultados deste estudo sugerem haver diferença significativa entre as médias de sensibilidade nas formas não exsudativa e exsudativa $(\mathrm{p}<0,05)$. Olhos com exsudação têm, em média, pior sensibilidade que olhos sem exsudação. Outros estudos registraram a presença de escotomas relativos e absolutos nas áreas de neovascularização ${ }^{(6-8)}$. A duração dos

Tabela 2. Média da sensibilidade dos locais com as formas não exsudativa e exsudativa de DMRI nos pacientes do grupo de casos

$\begin{array}{lcccc}\text { Formas } & \mathbf{n} & \text { Média (dB) } & \text { DP (dB) } & \text { C V } \\ \text { Não exsudativa } & 225 & 17,90 & 2,25 & 0,13 \\ \text { Exsudativa } & 80 & 14,50 & 5,69 & 0,39\end{array}$

$\mathrm{n}=$ número de locais com uma determinada forma de DMRI no grupo de casos $t=5,21 ; p<0,001^{*}\left({ }^{*}\right.$ significativo) sintomas e o tamanho da membrana neovascular foram associados à profundidade do escotoma ${ }^{(6)}$.

Uma vez que a área de neovasos é o local de maior diminuição de sensibilidade e a região vizinha apresenta melhor sensibilidade, convém buscar tratamentos focais, bem direcionados para essas áreas de lesão, evitando destruir áreas vizinhas. Nesse contexto, o tratamento de vasos nutridores e a terapia fotodinâmica parecem ser formas terapêuticas promissoras, promovendo uma destruição mais seletiva da região de neovascularização ${ }^{(9)}$. Várias pesquisas mostraram que tratamentos a laser com aplicações dispersas na região de neovascularização não apresentam resultados melhores que a evolução natural da doença de neovascularização ${ }^{(10-11)}$.

Os resultados aqui descritos parecem indicar que a forma exsudativa apresenta maior variação de sensibilidade do que a forma não exsudativa. Portanto, ao classificar um paciente em forma exsudativa utilizando somente o aspecto anatômico, pode-se realizar um julgamento incompleto, já que todos os casos com membrana neovascular podem ser incluídos na categoria de exsudativos, embora alguns tenham melhor sensibilidade que outros, o que sugere que se encontram em diferentes fases de evolução da DMRI, portanto com diferentes prognósticos. Áreas com neovasos e escotomas absolutos poderiam ser tratadas com fotocoagulação a laser, enquanto para lesões com escotomas relativos seria mais indicado o tratamento seletivo de vasos nutridores, terapia fotodinâmica ou cirurgia de translocação macular.

Pacientes com DMRI podem permanecer com a função macular na área ocupada pelo neovaso após cirurgia de remoção dessa membrana neovascular ${ }^{(12-13)}$.

Esses autores afirmam, entretanto, que, na maioria dos casos, após cirurgia para retirada de membrana neovascular, não há melhora da sensibilidade macular e registra-se um resultado visual ruim. O estudo anatomopatológico de membranas neovasculares sub-foveais retiradas cirurgicamente mostrou a presença de fotorreceptores, o que evidencia dano à função retiniana pós-cirurgia ${ }^{(14)}$. A maior variação de sensibilidade nos casos exsudativos evidenciada neste estudo sugere que no pré-operatório, baseado na função macular, há vários tipos de membrana neovascular, o que talvez justifi-

\begin{tabular}{|lcc|}
\hline \multicolumn{2}{|l|}{ Tabela 3. Comparação entre os diversos tipos de lesões anatômicas em relação às áreas com ausência de lesões anatômicas do grupo de casos } \\
\hline Variáveis & Diferença das & médias de sensibilidade (dB) \\
\hline ALA X Drusas duras & 0,94 & 0,462 \\
ALA X Drusas moles & 1,03 & 0,481 \\
ALA X Hiperpigmentação & $-0,07$ & 0,965 \\
ALA X Drusas duras + hiperpigmentação & 0,78 & 0,525 \\
ALA X Drusas moles + hiperpigmentação & 1,46 & 0,270 \\
ALA X Neovasos & 4,52 & $0,005^{*}$ \\
ALA X DEP & 3,31 & 0,088 \\
ALA X Neovasos + DEP & 4,31 & 0,095 \\
\hline ALA X Hipopigmentação e atrofia coriorretiniana & 9,18 & $<0,001^{*}$ \\
\hline ALA = ausência de lesões anatômicas; DEP= descolamento do epitélio pigmentado da retina & \\
*Significativo; teste de Fisher PLSD para a diferença de médias de sensibilidade & \\
\hline
\end{tabular}




\begin{tabular}{|c|c|c|c|}
\hline Comparação & $\mathbf{n}$ & Média & DP \\
\hline Controles & 372 & 20,42 & 1,10 \\
\hline Ausência de lesões anatômicas & 45 & 18,81 & 1,55 \\
\hline $\begin{array}{l}\mathrm{n}=\text { número de locais testados } \\
\mathrm{t}=6,77 ; \mathrm{p}<0,001^{*}\left({ }^{*} \text { significativo) }\right.\end{array}$ & & & \\
\hline
\end{tabular}

que os resultados variáveis nas cirurgias sub-maculares, embora a melhor ou pior sensibilidade pré-operatória não prediz o resultado visual pós-operatório ${ }^{(13)}$. Ressalta-se a importância da perimetria com o ORL no planejamento cirúrgico para retirada de membranas neovasculares subfoveais ${ }^{(15)}$.

Corroborando os achados de outros autores, os dados desta pesquisa não evidenciaram diferença na sensibilidade relacionada à presença de drusas, sugerindo que elas sejam marcadores de doença difusa do EPR, como demonstrado pela histopatologia ${ }^{(16-19)}$.

Os fotorreceptores são dependentes da habilidade do EPR para reciclar materiais essenciais para sua renovação e eliminar os resíduos. Como as drusas provavelmente refletem, em parte, a falha de enzimas degradativas, seria esperado algum dano à função dos fotorreceptores sobre a região das drusas $^{(20)}$. Entretanto, a sensibilidade macular parece ser a mesma em áreas com e sem drusas ${ }^{(17)}$.

Não foi observada diferença de sensibilidade sobre drusas moles, entretanto encontrou-se diferença de sensibilidade retiniana na área de drusas grandes utilizando o ORL ${ }^{(21)}$. Tais autores definiram drusas moles como aquelas com margens mal definidas e drusas grandes como aquelas com margens bem definidas, ambas acima de $125 \mu \mathrm{m}$. Esses autores estudaram quatro indivíduos sadios com idade entre 26 e 29 anos para testar a reprodutibilidade do exame de perimetria com ORL. Ao analisarem a sensibilidade retiniana em decibéis, verificaram que a variação encontrada nas cinco medidas de perimetria nesse grupo jovem era menor ou igual a $3 \mathrm{~dB}$. Definiram, assim, a diferença maior que $5 \mathrm{~dB}$ como ponto de corte para avaliar a variação entre as áreas com e sem drusas em pacientes com DMRI. Drusas maiores que $125 \mu \mathrm{m}$, bem delimitadas, tendem a evoluir mais freqüentemente para atrofia, embora todos os tipos de drusas possam evoluir para atrofia $^{(22-23)}$. É difícil a separação dos efeitos na sensibilidade decorrentes das drusas e da atrofia do EPR, uma vez que ambos podem ocorrer no mesmo local e podem representar estágios da evolução de um mesmo processo ${ }^{(17)}$. Esses autores provavelmente utilizaram áreas de atrofia nesse grupo de drusas grandes, pelas características anatômicas descritas e pelo resultado de escotomas absolutos (zero dB), encontrados em algumas áreas, demostrando atrofia retiniana nesse local. Estas importantes diferenças na metodologia e na interpretação dos resultados utilizados por outros autores ${ }^{(21)}$ podem justificar a diferença de seus resultados em relação aos encontrados neste estudo. Há também relatos de diminuição da sensibilidade em olhos com drusas, porém esse grupo, reconhecendo a limitação do método de perimetria utilizado (Humphrey 10-2), não sugeriu a correlação direta das drusas com a diminuição de sensibilidade, preferindo atribuir tal diminuição a um processo difuso que acomete EPR e retina sensorial.

No grupo de casos deste estudo não foi observada diferença significativa entre áreas aparentemente normais e áreas com hiperpigmentação, assim como no trabalho publicado por outro grupo ${ }^{(16)}$, embora tais dados não possam ser comparados diretamente pelas diferenças metodológicas já explicadas. Foram resultados diferentes dos encontrados por outros pesquisadores ${ }^{(25)}$, que descreveram menor sensibilidade nas áreas de hiperpigmentação, mas que também não podem ser comparados diretamente, uma vez que os autores utilizaram um programa de perimetria diferente, com parâmetros de luminosidade diferentes e em população diferente (altos míopes).

Utilizou-se método de avaliação de função macular e população de estudo diferentes das utilizadas neste estudo, encontrou-se diminuição da função macular relacionada à hiperpigmentação, drusas e atrofia ${ }^{(26)}$. Esses autores consideraram drusas com hipopigmentação e bordas bem definidas como drusas, e não atrofia, e referem que dos dez olhos com hiperpigmentação, nove estavam associados a atrofia na região testada, implicando uma quase perfeita correspondência entre essas duas lesões anatômicas no local testado. Drusas em regressão são mais esbranquiçadas e mais duras, na aparência, associadas a hiperpigmentação ${ }^{(22)}$. Ocasionalmente, as drusas podem regredir apenas com o sinal discreto de atenuação do EPR. A maioria dos casos de atrofia geográfica do EPR se desenvolve em áreas relacionadas a drusas em regressão. Supõe-se que tais fatos, além da não utilização do ORL, que permite a correlação anatômico-funcional direta, sejam fatores importantes para explicar a diferença entre os resultados encontrados.

As áreas com neovasos ou atrofia apresentaram sensibilidade significantemente diferente em relação às áreas com ausência de lesões anatômicas nos pacientes portadores de DMRI. Esse dado é concordante com outros pesquisadores ${ }^{(17)}$, que relataram diminuição da sensibilidade nas formas avançadas de DMRI, atrófica e exsudativa, embora esses autores tenham analisado a função de bastonetes e questionado a semelhança dos dados, se estudados os cones, como foi feito nesta pesquisa. Embora as áreas com neovasos e DEP e somente com DEP não tenham apresentado média de sensibilidade inferior à média da sensibilidade das áreas com ausência de lesões anatômicas, supõe-se que este resultado seja decorrente do número limitado desses casos na amostra estudada.

A evidência demonstrada neste estudo sobre a associação entre o comprometimento da sensibilidade macular e a presença de neovasos ou atrofia não implica que os resultados deste teste de função macular possam ser utilizados como fatores de risco para o desenvolvimento de DMRI. Recomenda-se a realização de estudos prospectivos, feitos a partir destes achados, para validar a utilização destes testes funcionais no prognóstico dos pacientes com DMRI. 
Nos outros tipos de lesões anatômicas (drusas duras, drusas moles, hiperpigmentação e DEP) de pacientes com DMRI, a perda de sensibilidade não foi significativa em relação às áreas sem lesões anatômicas ( $\mathrm{p}>0,05)$, evidenciando que essas lesões não seriam fatores individuais de piora da sensibilidade macular. Nessas lesões há um melhor potencial de benefício para tratamentos futuros.

Áreas sem lesões anatômicas, portanto aparentemente normais, no grupo de casos já apresentavam diminuição da sensibilidade retiniana em relação ao grupo de controles. Anormalidades da função visual podem estar presentes antes do aparecimento de atrofia visível pela oftalmoscopia na área foveal $^{(27)}$. De forma similar, outros grupos ${ }^{(28)}$ relatam diminuição da função foveal em pacientes com DMRI e acuidade visual de 20/20 sem nenhuma alteração anatômica detectável pela oftalmoscopia ou fotografia do fundo ocular. Isso sugere que os métodos de avaliação anatômica "in vivo", atualmente utilizados, são insuficientes para detectar alterações bioquímicas, possivelmente herdadas, que ocorrem nas fases precoces da doença ${ }^{(29)}$.

Neste estudo foi utilizado o ORL para realizar a correlação entre as alterações funcionais e alterações anatômicas em pacientes com DMRI. A medida da sensibilidade macular pode acrescentar dados à avaliação anatômica e no futuro essas medidas específicas poderão ser utilizadas para a melhor classificação dos pacientes com DMRI, como fatores de risco ou medidas do resultado de novos tratamentos.

\section{CONCLUSÕES}

O estudo da sensibilidade luminosa na área macular evidenciou:

1) Nas lesões anatômicas classificadas como drusas duras, drusas moles, hiperpigmentação, hipopigmentação e descolamento do epitélio pigmentado, observou-se perda de sensibilidade, porém não significativa em relação às áreas com ausência de lesões anatômicas nos pacientes portadores de DMRI, sugerindo que essas lesões não seriam fatores individuais de piora da sensibilidade;

2) As áreas com neovasos ou atrofia apresentaram sensibilidade significantemente diferente em relação às áreas com ausência de lesões anatômicas nos pacientes portadores de DMRI;

3) Houve perda funcional significativa em áreas com ausência de lesões anatômicas nos pacientes portadores de DMRI em relação à sensibilidade nos indivíduos sem DMRI.

\section{ABSTRACT}

Purposes: To evaluate the correlation between anatomical changes (hard druses, soft druses, hyperpigmentation, new vessels, detachment of retinal pigment epithelium, hypopigmentation and chorioretinal atrophy) and light sensitivity in patients with age-related macular degeneration (ARMD); analyze macular sensitivity in areas with no anatomical lesions in patients with ARMD and compared them to the control group in order to detect if there was any functional lesion in areas with no anatomical changes. Methods: A cross-sectional, comparative, descriptive and analytic study was performed. The case group consisted of 31 subjects with ARMD aged between 51 and 88 years. The control group consisted of 31 "healthy" subjects, without ARMD aged between 61 and 80 years. The groups were matched for gender and age. We performed static macular perimetry, red-red, using a scanning laser ophthalmoscope (SLO). Results of macular perimetry were correlated with the anatomic lesion identified in the same site by infrared laser and color photographs. Results: Areas with new vessels or atrophy showed a significantly different sensitivity in relation to areas without anatomical lesions in patients with ARMD. There was significant functional loss in areas with no anatomical lesions in patients with ARMD in relation to the control group. Conclusions: Areas with new vessels or atrophy could be distinct factors for worsening of the localized macular sensitivity. There might be functional loss even in areas with no apparent anatomical changes in ARMD patients.

Keywords: Perimetry; Ophthalmoscopes; Macular degeneration; Choroidal neovascularization; Aging factors

\section{REFERÊNCIAS}

1. Sunness JS, Massof RW, Johnson MA, Bressler NM, Bressler SB, Fine SL Diminished foveal sensitivity may predict the development of advanced agerelated macular degeneration. Ophthalmology. 1989;96(3):375-81.

2. Sunness JS, Schuchard RA, Shen N, Rubin GS, Dagnelie G, Haselwood DM. Landmark-driven fundus perimetry using the scanning laser ophthalmoscope. Invest Ophthalmol Vis Sci. 1995;36(9):1863-74.

3. Timberlake GT, Mainster MA, Webb RH, Hughes GW, Trempe CL. Retinal localization of scotomata by scanning laser ophthalmoscopy. Invest Ophthalmol Vis Sci. 1982;22(1):91-7.

4. Timberlake GT, Van de Velde FJ, Jalkh AE. Clinical use of scanning laser ophthalmoscope retinal function maps in macular disease. Lasers Light Ophthalmol. 1989;2:211-22.

5. Acosta F, Lashkari K, Reynaud X, Jalkh AE, Van de Velde F, Chedid N. Characterization of functional changes in macular holes and cysts. Ophthalmology. 1991;98(12):1820-3.

6. Schneider U, Turner KL, Inhoffen W, Kreissig I. Scanning laser microperimetry of well-defined choroidal neovascularization secondary to age-related macular degeneration. Lasers Light Ophthalmol. 1996;7:167-72.

7. Schneider U, Inhoffen W, Gelisken F, Kreissig I. Assessment of visual function in choroidal neovascularization with scanning laser microperimetry and simultaneous indocyanine green angiography. Graefes Arch Clin Exp Ophthalmol. 1996;234(10):612-7.

8. Tezel TH, Del Priore LV, Flowers BE, Grosof DH, Benenson IL, Zamora RL, Kaplan HJ. Correlation between scanning laser ophthalmoscope microperimetry and anatomic abnormalities in patients with subfoveal neovascularization. Ophthalmology. 1996;103(11):1829-36.

9. Shiraga F, Ojima Y, Matsuo T, Takasu I, Matsuo N. Feeder vessel photocoagulation of subfoveal choroidal neovascularization secondary to age-related macular degeneration. Ophthalmology. 1998;105(4):662-9. Comment in: Ophthalmology. 1998;105(12):2163-4. Ophthalmology. 1998;105(12):2164-5.

10. Arnold J, Algan M, Soubrane G, Coscas G, Barreau E. Indirect scatter laser photocoagulation to subfoveal choroidal neovascularization in age-related macular degeneration. Graefes Arch Clin Exp Ophthalmol. 1997;235(4):208-16.

11. Cardillo Piccolino F, Ghiglione D, Allegri P. Grid laser treatment of occult choroidal neovascularization in age related macular degeneration. Int Ophthalmol. 1993;17(2):77-83. 
12. Loewenstein A, Sunness JS, Bressler NM, Marsh MJ, de Juan E Jr. Scanning laser ophthalmoscope fundus perimetry after surgery for choroidal neovascularization. Am J Ophthalmol. 1998;125(5):657-65

13. Tsujikawa M, Tsujikawa K, Lewis JM, Tano Y. Change in retinal sensitivity due to excision of choroidal neovascularization and its influence on visual acuity outcome. Retina. 1999;19(2):135-40.

14. Oréfice JL, Miranda D, Aguiar AP, Nehemy MB. Estudo anatomopatológico de membranas neovasculares subretinianas. Arq Bras Oftalmol. 1998;61:505.

15. Hudson HL, Frambach DA, Lopez PF. Relation of the functional and structural fundus changes after submacular surgery for neovascular age-related macular degeneration. Br J Ophthalmol. 1995;79(5):417-23.

16. Midena E, Degli Angeli C, Blarzino MC, Valenti M, Segato T. Macular function impairment in eyes with early age-related macular degeneration. Invest Ophthalmol Vis Sci. 1997;38(2):469-77.

17. Sunness JS, Johnson MA, Massof RW, Marcus S. Retinal sensitivity over drusen and nondrusen areas. A study using fundus perimetry. Arch Ophthalmol. 1988;106(8):1081-4.

18. Sunness JS, Massof RW, Bressler NM, Bressler SB. S cone pathway sensitivity in eyes with high risk and low risk drusen characteristics. Applied Optics. 1989;28(6):1158-64

19. Van de Velde FJ, Jalk AE, Shamas H. High illumination microperimetry of drusen related maculopathy. Invest Ophthalmol Vis Sci. 1992;33:1229.

20. Pauleikhoff D, Harper CA, Marshall J, Bird AC. Aging changes in Bruch's membrane. A histochemical and morphologic study. Ophthalmology. 1990;97 (2):171-8.
21. Takamine Y, Shiraki K, Moriwaki M, Yasunari T, Miki T. Retinal sensitivity measurement over drusen using scanning laser ophthalmoscope microperimetry. Graefes Arch Clin Exp Op hthalmol. 1998;236(4):285-90.

22. Sarks JP, Sarks SH, Killingsworth MC. Evolution of soft drusen in agerelated macular degeneration. Eye. 1994;8(Pt 3):269-83.

23. Sarks JP, Sarks SH, Killingsworth MC. Evolution of geographic atrophy of retinal pigment epithelium. Eye. 1988;2(Pt 5):552-77.

24. Midena E, Segato T, Blarzino MC, Degli Angeli C. Macular drusen and the sensitivity of the central visual field. Doc Ophthalmol. 1994;88(2):179-85.

25. Chen JF, Elsner AE, Burns SA, Hansen RM, Lou PL, Kwong KK, Fulton AB. The effect of eye shape on retinal responses. Clin Vision Sci. 1992;7(6): 521-30.

26. Eisner A, Stoumbos VD, Klein ML, Fleming SA. Relations between fundus appearance and function. Eyes whose fellow eye has exudative age-related macular degeneration. Invest Ophthalmol Vis Sci. 1991;32(1):8-20. Erratum in: Invest Ophthalmol Vis Sci 1991;32(5):1507.

27. Sunness JS, Gonzalez-Baron J, Applegate CA, Bressler NM, Tian Y, Hawkins $\mathrm{B}$, et al. Enlargement of atrophy and visual acuity loss in the geographic atrophy form of age-related macular degeneration. Ophthalmology. 1999;106 (9):1768-79.

28. Eisner A, Fleming SA, Klein ML, Mauldin WM. Sensitivities in older eyes with good acuity: eyes whose fellow eye has exudative AMD. Invest Ophthalmol Vis Sci. 1987;28(11):1832-7.

29. Souza EC. Degeneração macular relacionada com a idade (D.M.R.I.) considerações clínico-patológicas recentes. Arq Bras Oftalmol. 1995;58:254 